\title{
Optimization of culture conditions of soymilk for equol production by Bifidobacterium breve 15700 and Bifidobacterium longum BB536
}

\begin{abstract}
This study analyzed the effect of $\mathrm{pH}(\mathrm{X} 1)$, temperature (X2) and inulin amount (X3) on transformation of isoflavones (daidzin and daidzein) to equol in soymilk fermented with Bifidobacterium spp. All responses significantly $(\mathrm{p}<0.05)$ fitted into quadratic models with coefficients of determination (R2) close to $1(0.935-0.989)$. At $24 \mathrm{~h}$ of fermentation, amounts of daidzin and daidzein were influenced by all factors. While at $48 \mathrm{~h}$, all factors affected daidzin and only temperature affected daidzein. Equol production was influenced by $\mathrm{pH}$ and temperature in $24 \mathrm{~h}$ and by all factors in $48 \mathrm{~h}$ fermentation. The optimum conditions for equol production were $\mathrm{pH} 8,30^{\circ} \mathrm{C}$ and $0.5 \%$ inulin. Model validation demonstrated there was no significant $(\mathrm{p}>0.05)$ difference between the experimental and predicted values, suggested the suitability of established models in explaining the daidzin and daidzein transformation to equol as a function of $\mathrm{pH}$, temperature and inulin.
\end{abstract}

Keyword: Equol; Bifidobacterium; Inulin; Transformation; Daidzin; Daidzein 